\title{
Potential Protection by Antioxidants of the Action of Tobacco Smoke on the Metabolism of Cultured Bovine Lenses
}

\author{
Bormusov $E^{*}$, Reznick $A Z$ and Dovrat $A$
}

Rappaport Faculty of Medicine, Technion-Israel Institute of Technology, Haifa, Israel

\begin{abstract}
Purpose: Smoke from cigarette smoking (CS) has been proposed to be a major environmental risk factor for a variety of human diseases and was implicated also in cataract, an eye lens pacification, which is a major cause of blindness. We have undertaken a study to investigate the effect of smoke on the physiological integrity and metabolism of organ cultured lenses. Lenses in organ culture are metabolically active and have functional defense systems, thus they provide an appropriate model for studying the effects of smoke. Also the possible protective action of $\mathrm{N}$-acetylL-cysteine (NAC) which is a precursor of glutathione and the iron chelators Deferoxamine (DFO), was estimated as potential protective agents against cataract.
\end{abstract}

Methods: Bovine lenses were incubated in organ culture conditions at $35^{\circ} \mathrm{Cfor} 6$ days. Treated lenses were 4 day in the culturally environment daily sated with a cigarette smoke under various doses of pressure-250,500 psi. Two of the experimental groups were treated with NAC $(1 \mathrm{mM})$ and DFO $(2.5 \mu \mathrm{g} / \mathrm{ml})$ as antioxidants. An automated scanning laser system was used for daily testing of both treated and control lenses. At the end of the culture period, lenses were analyzed by inverted microscopy. We have used preparations of the forward monolayer epithelium bovine lenses from all experiments. For this purpose the capsule opened and crystalline lens fibers were cleaned. On the object-plate them there was only a capsule and a cellular monolayer epithelium. Changes of morphology of cells and the contents of the nucleic acids was estimated using the Einarson - DNA-RNA staining method. Reactive Oxygen Species (ROS) were estimated with 5- (and 6-) chloromethyl-2',7'-dichloro-dihydrofluorescein diacetate, acetyl ester (CM-H2DCFDA, C6827) to measure the level of cellular oxidation in the cells of lens epithelium. The levels of ROS were measured by monitoring the fluorescent intensity relative to that of control cultures under fluorescent microscopy. Nucleic acids were stained with Propidium lodide.

Results: Exposure of cigarette smoke in cultured medium under negative pressure, optical quality of lenses and the structural changes were demonstrated by decreased light transmission, increase in focal length variability and a decrease in morphological integrity such as hyperplasia and hypertrophy of epithelial cells. The group exposed to NAC and to DFO, demonstrated reduced optical changes representing smaller lens injury. The lenses show almost no volume changes. However, the baseline fluorescence of controls varied between experiments. A dose-dependent increase in ROS generation in cultures was also evident.

Conclusions: We have shown that increasing the amount CS exposure, for a relatively short time, causes a sharp increase in the damage to the lens. Smoking is an independent risk factor that has dose-response effects. It causes morphological and functional changes to the lens. Based on the independent effects of NAC and DFO, we propose to possibly use them as means of prevention and/or treatment of cataract in heavily smoking people.

\section{Introduction}

Cigarette smoking (CS) has been implicated in the pathogenesis of cataract, but the pathogenic mechanism by which cigarette smoke causes cataract is yet to be elucidated. Whether there is a distinct direct dose-dependent correlation between cigarette smoke exposure and risk is debatable, as some experimental clinical studies have shown a nonlinear relation to cigarette smoke exposure. Experimental and clinical data support the hypothesis that cigarette smoke exposure increases oxidative stress as a potential mechanism for initiating cardiovascular dysfunction [1]. The effects of CS on immunity are far-reaching and complex; both pro-inflammatory and suppressive effects may be induced. The net effect of CS on immunity depends on many variables, including the dose and type of tobacco, the route and chronicity of exposure, and the presence of other factors at the time of immune cell stimulation, such as Toll receptor ligands or other inflammatory mediators. CS impairs innate defenses against pathogens, modulates antigen presentation, and promotes autoimmunity. CS also impairs immunity in the oral cavity and promotes gingival and periodontal disease and oral cancer. The recognition of specific mechanisms by which CS affects host immunity is an important step toward elucidating mechanisms of tobacco-induced disease and may identify novel therapeutic approaches for the management of diseases that afflict smokers [2]. There has been suggestion that oxidative damage caused by accumulation of Fenton reagents (iron and copper) in the lens can cause lens damage and possibly cataract [3]. The lenses of experimental animals (Wistar rats) showed significantly decreased zinc and increased iron, and calcium concentration relative to those of sham exposed controls. However, no significant difference was found in the copper contents of the lenses. Distinct histopathological changes such as hyperplasia of epithelial cells and elevations of calcium concentration detected in the lenses of experimental group animals suggested that the lens damage was a result of in-vivo exposure to tobacco smoke. The

*Corresponding author: Bormusov E, Rappaport Faculty of Medicine, Technion - Israel Institute of Technology, Haifa, Israel, E-mail: bormusov@tx.technion.ac.il

Received June 01, 2013; Accepted August 27, 2013; Published September 04, 2013

Citation: Bormusov E, Reznick AZ, Dovrat A (2013) Potential Protection by Antioxidants of the Action of Tobacco Smoke on the Metabolism of Cultured Bovine Lenses. Metabolomics 3: 124. doi:10.4172/2153-0769.1000124

Copyright: (c) 2013 Bormusov E, et al. This is an open-access article distributed under the terms of the Creative Commons Attribution License, which permits unrestricted use, distribution, and reproduction in any medium, provided the original author and source are credited. 
authors propose that increased metal contents in the lens can cause lens damage by the mechanism of oxidative stress through formation of oxygen radicals via metal catalyses Fenton reaction. Cataract and age-related macular degeneration (AMD) are the major causes of vision impairment and blindness worldwide [4]. Both conditions are strongly age related with earlier signs (usually asymptomatic) occurring in middle age and becoming more severe and more prevalent with increasing age. The aetiology of these conditions is thought to fit with the 'free radical theory' of ageing which postulates that ageing and age-related diseases result from the accumulation of cellular damage from reactive oxygen species (ROS). Mitochondrial energy production is a major source of endogenous ROS. External sources of ROS include environmental sources especially solar radiation, biomass fuels and tobacco smoking. There is strong evidence from epidemiological studies that smoking is a risk factor for both cataract and AMD. There is moderate evidence for an association with sunlight and cataract but weak evidence for sunlight and AMD. The few studies that have investigated this suggest an adverse effect of biomass fuels on cataract risk. The antioxidant defence system of the lens and retina include antioxidants vitamins $\mathrm{C}$ and $\mathrm{E}$ and the carotenoids lutein and zinc, and there is mixed evidence on their associations with cataract and AMD from epidemiological studies. Weiner et al. [5] study was to elucidate the effect of cigarette smoke (CS) on nitrotyrosine formation in saliva proteins. They exposed saliva to CS, in vitro, and used Western Blotting (WB) and monoclonal anti-nitrotyrosine antibody to assess the level of saliva protein nitration. As saliva contains extensive amounts of nitrites, it was no surprise that at basal levels, saliva proteins, albumin, and alpha-amylase all were already nitrated. The WB also revealed that with continuous exposure to CS the tyrosine nitration of both albumin and $\alpha$-amylase is declining significantly after $3 \mathrm{~h}$. A quite similar effect was seen after exposure to aldehydes, but to a less extent as compared to CS. Exposure of nitrotyrosine-modified bovine serum albumin (BSA-N) to aldehydes, produced a similar effect, meaning a decrease in tyrosine nitration. Therefore, it is possible that the main player in the CS smoke denitration effect on salivary proteins is the aldehyde group and not the double bond of unsaturated aldehydes, masking the nitro group and thus not recognized by the antinierotyrosine antibodies.

Exposure of human plasma in vitro to gas-phase cigarette smoke (CS) causes a marked modification of plasma proteins as measured by protein carbonyl assay [6]. Aldehydes present in CS may cause this elevation of protein carbonyls by reacting with sulfhydryl groups of proteins. Addition of $1 \mathrm{mM}$ of GSH and $\mathrm{N}$-acetylcysteine considerably protected LDH and amylase activities, suggesting that sulfhydryl groups are affected in $\mathrm{LDH}$ and amylase. On the other hand, addition of $1 \mathrm{mM}$ ascorbate caused a further loss of LDH and amylase activities, which could be partially prevented by the addition of desferrioxamine mesylate, implicating metal-catalyzed oxidation processes increased by the addition of ascorbate.

Khanna et al. [7] demonstrated that chronic tobacco smoke exposure leading up to acute MI in rats is associated with greater histological extent of myocardial necrosis and consequent worse LV function. These findings are associated with increased transcriptomic expression of pro-inflammatory cytokines, tissue repair molecules and markers of oxidative stress in the myocardium. The results demonstrated that an N-acetyl cysteine (NAC) treatment significantly reduced tobacco-exposed induced infarct size and percent fractional shortening. Decreased intracardiac mRNA expression of the markers of inflammation, tissue repair and oxidative stress and circulating levels of pro-inflammatory cytokines accompanied these positive effects of NAC.
The ocular lens is a unique tissue with a distinctive cellular architecture. The lens consists of two cell types, the single layer of lens epithelial cells on its anterior surface, which are responsible for the growth and development of the entire lens, and the differentiated lens fiber cells, with elongated morphology that do not turn over their proteins throughout life. Lens epithelial cells provide metabolic support to the entire lens, and are also the first cells in the lens to be exposed to damage. Moreover, the epithelium is the site at which metabolic enzymes and transport systems are concentrated, thus making these cells essential for maintaining lens homeostasis, and as the first line of defense against environmental damage. In the present investigation we explored the interactions of cigarette smoke with lens constituents in an attempt to characterize some potential mechanisms by which cigarette smoke interacts with antioxidants and how this may contribute. Desferrioxiamine (Desferal $\left.{ }^{\circledR}, \mathrm{DFO}\right)$ is a selective high affinity iron chelator, rendering iron as a stable ferric complex [8]. DFO has been often used as a means of reducing tissue oxidative stress and injury. Reddan et al. [8] demonstrated that DFO protects cultured rabbit lens epithelial cell from oxidative insult. Atkuri [9] showed the effectiveness of DFO in preventing cataractous changes in rat lenses following in vivo exposure of rats to oxidative stress.

We have used an additional alternative strategy for curbing ROS-induced injury and protection of the lens. This involved the employment of $\mathrm{N}$-acetyl cysteine (NAC), to scavenge free radicals [10] and to replenish reduced sulfhydryl residues . NAC is a precursor of glutathione - the major source of cellular sulfhydryl groups and acts as a potent an anti-inflammatory agent. The aim of our study was to determine the relative effectiveness of various antioxidants in ameliorating the adverse effects of cigarette smoke on the lens and epithelium cells.

\section{Materials and Methodology}

\section{Lenses and organ culture system}

Eyes were obtained from 1-year-old male calves at an abattoir. Lenses were dissected within 2-4 hours after enucleating under sterile conditions (Figure 1). Each lens was placed in a glass and silicon rubber chamber containing $24 \mathrm{ml}$ culture medium (M199 with Earl's balanced salt solution, supplemented with $5.96 \mathrm{~g} / \mathrm{l}$ HEPES, 3\% dialyzed fetal calf serum and the antibiotics penicillin $100 \mathrm{U} / \mathrm{ml}$ and streptomycin 0.1 $\mathrm{mg} / \mathrm{ml}$ ). The glass chamber was filled with sufficient culture medium to completely immerse the lenses (Figure 2).

The lenses were incubated at $35^{\circ} \mathrm{C}$. Culture medium was changed daily. Experimental treatments began after pre-incubation of 24 hours $[11,12]$. Bovine lenses were incubated in organ culture conditions for 6 days. Treated lenses were exposed to CS every day for 4 days at various doses for amount of smoke of pressure-250, $500 \mathrm{mmHg}$ (equivalent to 1-5 cigarettes) in a special device. To two of the experimental as exposed groups we have added NAC $(1 \mathrm{mM})$ and DFO $(2.5 \mu \mathrm{g} / \mathrm{ml})$ as antioxidants. Exposure to CS was performed by a system consisting of a
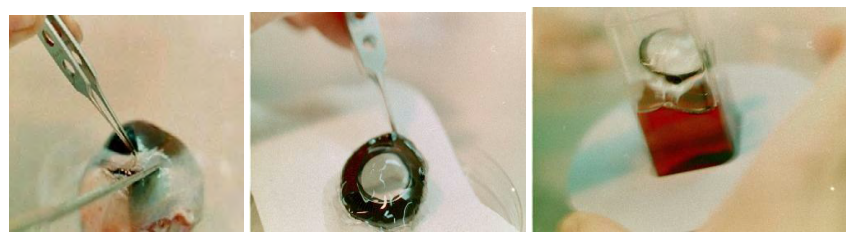

Figure 1: Lens extraction from a bovine eye 


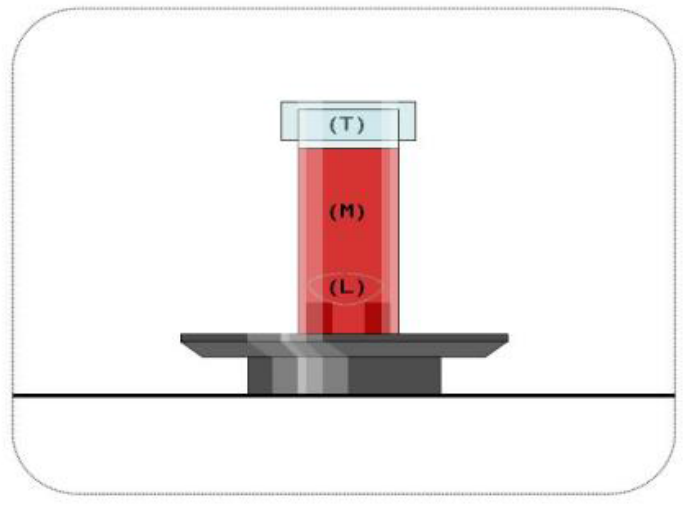

Figure 2: Lens container - a special test-tube.

chamber attached to a vacuum pump and a negative pressure gauge (up to $600 \mathrm{mmHg}$ ) at one end and a cigarette at the other end (Figure 3). Medium were placed inside the chamber. Then, the vacuum pump was activated, valve B was closed and valve A was opened until a desired level of negative pressure was created inside the chamber. By using the vacuum pump the pressure inside the chamber was reduced relatively to the atmospheric pressure outside. Subsequently, a TIME commercial cigarette containing $14 \mathrm{mg}$ of tar and $0.9 \mathrm{mg}$ of nicotine and filter (Dubek Ltd., Tel Aviv, Israel) was lit, valve A was closed and valve B between the burning cigarette and chamber was opened for 10 seconds allowing CS to enter the chamber. Creating reduced pressure inside the chamber allowed the drawing of CS from the burning cigarette into the chamber. Thus, the dose of CS entering the chamber equated the level of negative pressure created inside the chamber. Smoke passing through the cigarette filter was considered as vapor phase CS. After exposure to CS the chamber with the medium was sealed and transferred for different incubation times at $35^{\circ} \mathrm{C}[13]$.

\section{Optical monitoring system}

An optical bench [14] was used for daily testing of both exposed and control lenses. A $670 \mathrm{~nm}$ diode laser with the beam parallel to the axis of the lens was directed towards the cultured lens along one meridian in $0.5 \mathrm{~mm}$ increments. After passing through the lens, the laser beam is refracted and the system determines the back vertex focal length for every beam position. Each scan consists of measurements of the same beam from 22 different points across the lens. This optical monitoring apparatus uses a computer-operated scanning laser beam, a video-camera system and a video frame analyzer to record the focal length and transmittance of the cultured lens. The scanner is designed to measure the focal length at points across the diameter of the lens. The lens container permits the lens to be exposed to a vertical laser beam from below. The laser source projects its light onto a plain mirror, which is mounted at $45^{\circ} \mathrm{C}$ on a carriage assembly. The mirror reflects the laser beam directly up through the test lens. The mirror carriage is connected to a positioning motor, which moves the laser beam across the lens. The camera sees the cross section of the beams and, by examining the image at each position of the mirror, Scan-Tox software is able to measure the quality of the lens by calculating the back vertex distance for each beam position. A lens of good optical quality is able to focus the laser beam from the various locations at a fairly sharp focal point [15]. When the lens is damaged, it fails to intersect these laser beams at a consistent focal point. Measurements of variability of focal length are a quantitative measure of lens damage.

\section{Lens epithelium microscopy and histochemistry}

At the end of the culture period, lenses were analyzed by inverted microscopy. Lenses were photographed using an inverted microscope in order to assess damage at different depths of the lens. For the subsequent studies we have used epithelium preparations of a monolayer of bull lenses from all experiments. Faddeev's method [16] was used. For this purpose the used capsule was opened were cleaned and transparent fibers of a lens. On an object plate here was only a capsule and monolayer cellular epithelium (Figure 4).This was evaluated account the different grade of its capsule, i.e. topographical features of central intermediate and equatorial zone epithelium cells [17].

Treated lenses were analyzed morphologically and by assessment of the nucleic acid staining of the lens epithelium (Pearse's method [18] uses gallocyanin-chromalum by Einarsone with enzyme for specificity. The procedure includes fixation of the samples with methanol for $30 \mathrm{~min}$, incubation with the gallocyanin-chromalum solution for 48 hours. The chromalum $\left(\mathrm{K}_{2} \mathrm{SO}_{4} \cdot \mathrm{Cr}_{2}\left(\mathrm{SO}_{4}\right) 3 \cdot 24 \mathrm{H}_{2} \mathrm{O}\right)$ with gallocyanin at $\mathrm{pH}=1.64$ binds to nucleic acids and give it dark blue color. Reactive Oxygen Species (ROS) in epithelial cells lens were detected with 5(and 6-) chloromethyl-2',7'-dichlorodihydrofluorescein diacetate, acetyl ester (CM-H2DCFDA, C6827) measuring the level of cellular oxidation in the cells of lens epithelium. When oxidising the agent is added to the cells this reagent is converted to fluorescent isomer. The fluorescent signal was detected with a fluorescence microscope, using sources of excitement and filters, corresponding to fluorescein. Levels of ROS were measured by measuring fluorescent intensity of the cells in culture. Nuclei were labelled by Propidium Iodide. The Quantitative intensities of the histochemistry reactions in central and equatorial zones was be done using the program Image-Pro Plus, measuring Integrated Optical Density in each cell using 30-50 cells of each slide. A change is defined as significant if the difference between control and treated groups reaching value of $\mathrm{P}<0.05$.

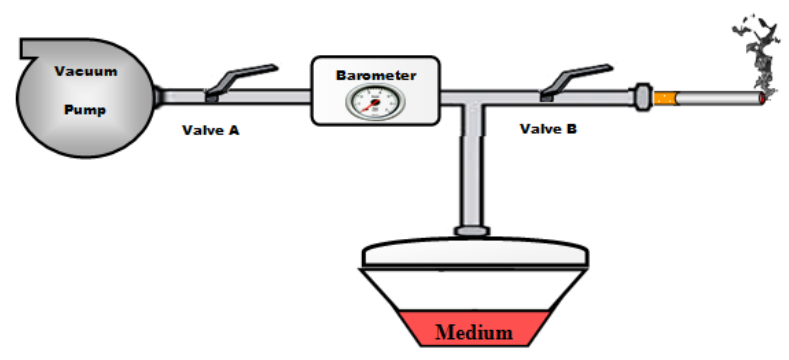

Figure 3: CS exposure apparatus (Rom et. al.,2013, with permission).

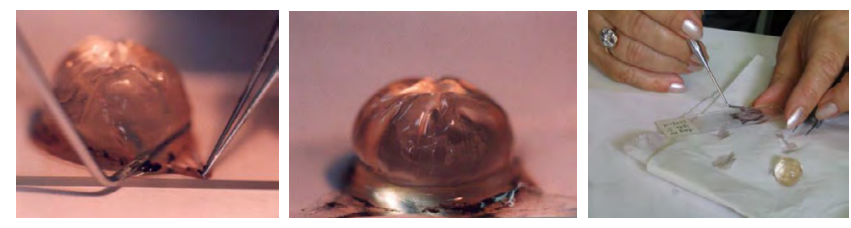

Figure 4: Opened a capsules of a lenses. 
Citation: Bormusov E, Reznick AZ, Dovrat A (2013) Potential Protection by Antioxidants of the Action of Tobacco Smoke on the Metabolism of Cultured Bovine Lenses. Metabolomics 3: 124. doi:10.4172/2153-0769.1000124

\section{Results}

\section{Optical analysis}

Lens optical quality was tested every 24 hours. Our optical system can measure minute changes in lens transparency as demonstrated by changes in focal length and transmittance. A $670 \mathrm{~nm}$ diode laser with the beam parallel to the axis of the lens was directed towards the cultured lens along one meridian in $0.5 \mathrm{~mm}$ increments. After passing through the lens, the laser beam is refracted and the system determines the back vertex focal length for every beam position. Each scan consists of measurements of the same beam from 22 different points across the lens. A lens of good optical quality is able to focus the laser beam from the various locations. When the lens is damaged its ability to focus the laser beam is altered. The blue line connects the points of the Back Vertex Distance for each ray passing through the lens. The pink line shows the relative intensity of each beam transmitted intensity normalized to the control lenses. Focal length variability represented the variation in the focal lengths of the 22 beams passed through the lens during each scan and was calculated as the standard error of the mean of the 22 focal lengths. Each point on the graph represents the average of the lenses of that treatment.

Control lenses (red) show almost no variation in focal length during the 6 days of the culture (Figure 5). On the other hand, lenses exposed to the different treatments show variation in focal length (damage) starting from about 48 hours after initial exposure to CS -250 and 500 $\mathrm{mmHg}$. For the CS treatment, damage can be observed on day 3. The lenses try to correct the damage (days 4-6). Damage increases on day 4 and repair occurs again from day 5 , then the damage increased again.

Lenses incubated with the CS and antioxidants retain their optical quality up to day 3-4 of the culture, but later focal length variability increased even more than in the treatment. Lenses incubated with CS and the antioxidant show damage one day later than those with DFO alone, but the NAC improve lens optical quality in terms of focal length variability.

\section{Inverted Microscopy photographs}

The results show that high concentration CS causes damage to lens epithelium and fibers and treatment with the antioxidants reduces the damage caused by CS levels (Figures 6-8).

\section{Nucleic acid staining of the lens epithelium cells}

Intact cells, each one with nucleus, with native. Visible changes in comparison with control it isn't observed (Figures 9 and 10).

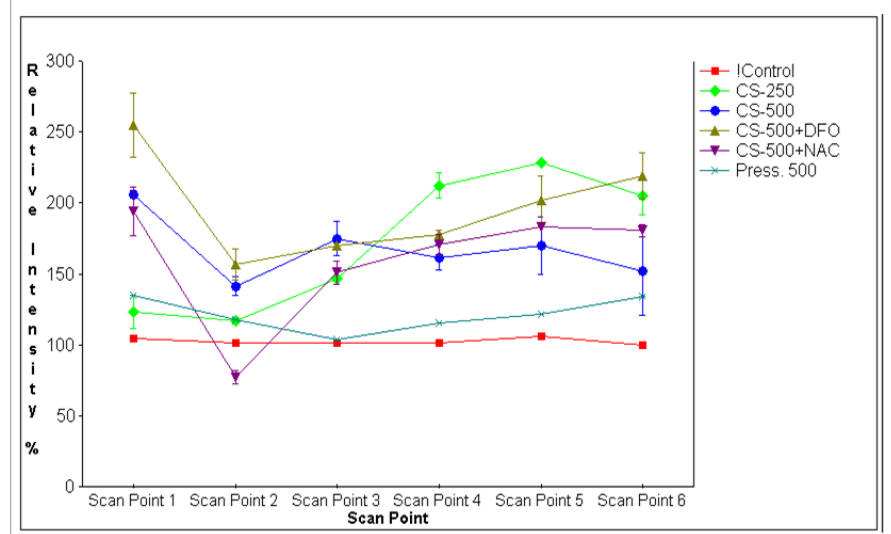

Figure 5: Day 6. Lens Optical Quality - Intensity.

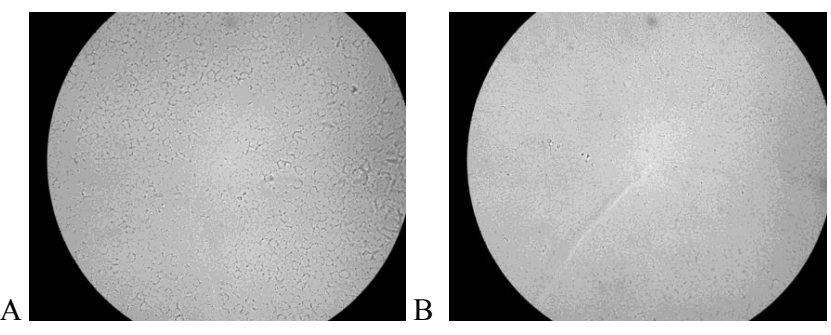

Figure 6: Inverted microscopy photograph of control lens(A), lens incubated with medium pressure $500 \mathrm{mmHg}(\mathrm{B})$.
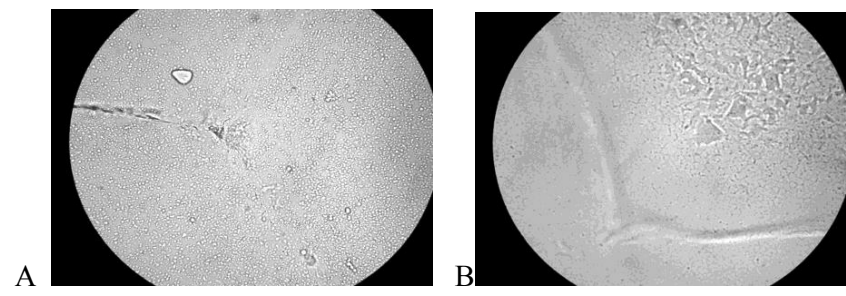

Figure 7: Inverted microscopy photograph lens incubated with medium CS$250 \mathrm{mmHg}(\mathrm{A})$, lens incubated with medium CS-500 $\mathrm{mmHg}(B)$.
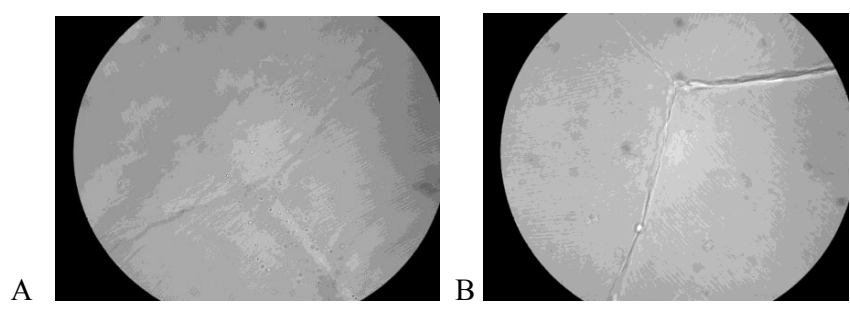

Figure 8: Inverted microscopy photographs lens incubated with medium CS$500 \mathrm{mmHg}+\mathrm{DFO}(\mathrm{A})$, lens incubated with medium CS-500 mmHg + NAC(B).
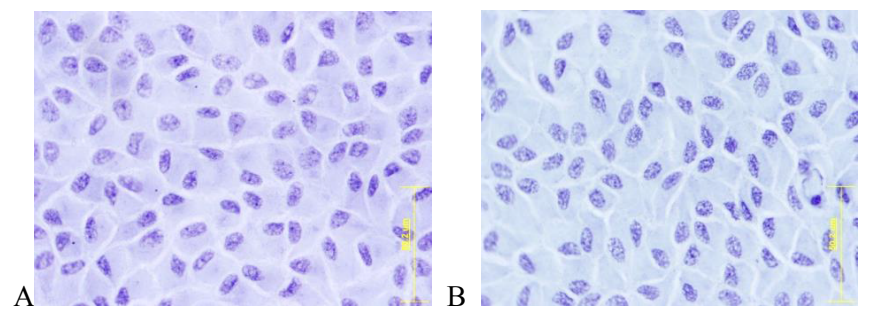

Figure 9: Control lens epithelium-center (A). Epithelium cells. Pressure 500 $\mathrm{mmHg}(\mathrm{B})$.

There is larger space between the cells and changes in organization after CS influence. Some cells are damaged without nucleus and no membrane. Cells organization shows integrity is disrupted, such as hyperplasia and hypertrophy of epithelial cells (Figure 11).

Antioxidants a little reduced the damage caused by the high maintenance of CS (Figures 12 and 13).

These graphics show that under the influence of CS the optical density of nucleic acids in cages of an epithelium varies. As the area of cages changes also. However, the DFO and NAC correct this shortcoming. 
Citation: Bormusov E, Reznick AZ, Dovrat A (2013) Potential Protection by Antioxidants of the Action of Tobacco Smoke on the Metabolism of Cultured Bovine Lenses. Metabolomics 3: 124. doi:10.4172/2153-0769.1000124

Page 5 of 7

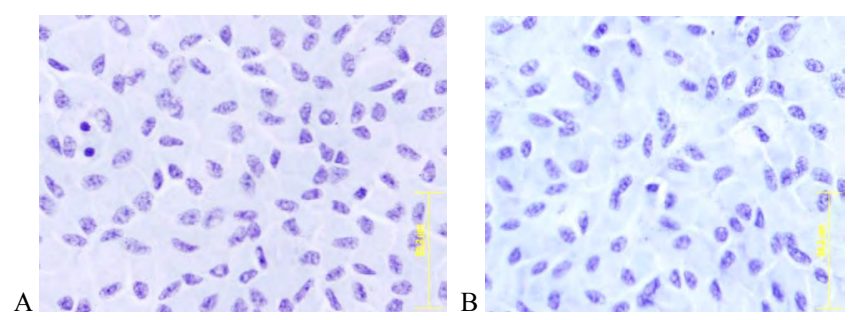

Figure 10: Epithelium cells. CS-250 (A). . Epithelium Cells. CS-500(B).

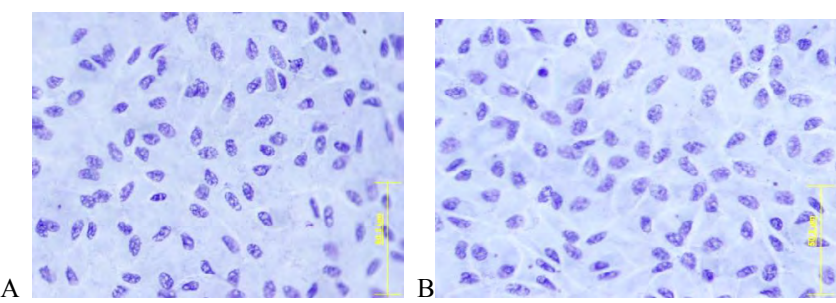

Figure 11: Epithelium cells. CS-500 with DFO (A). Epithelium cells. CS-500 with NAC (B).

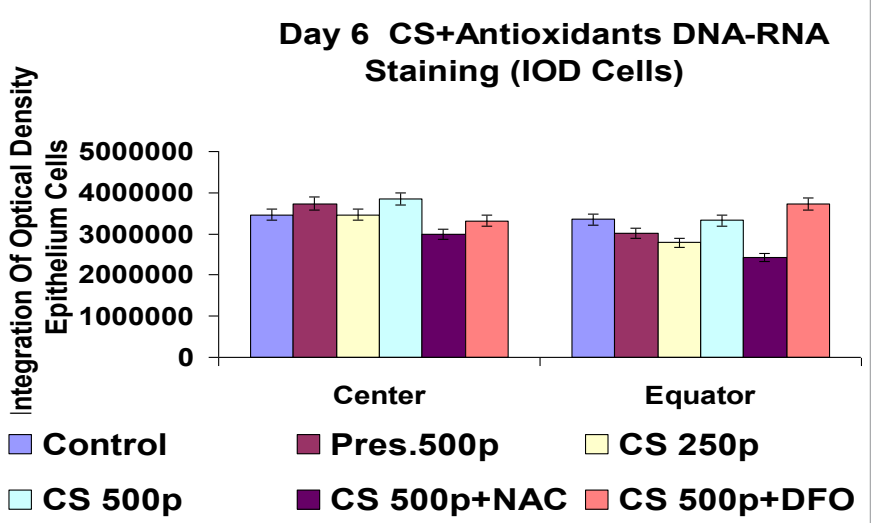

Figure 12: Integration of optical density epithelium cells. DNA/RNA staining.

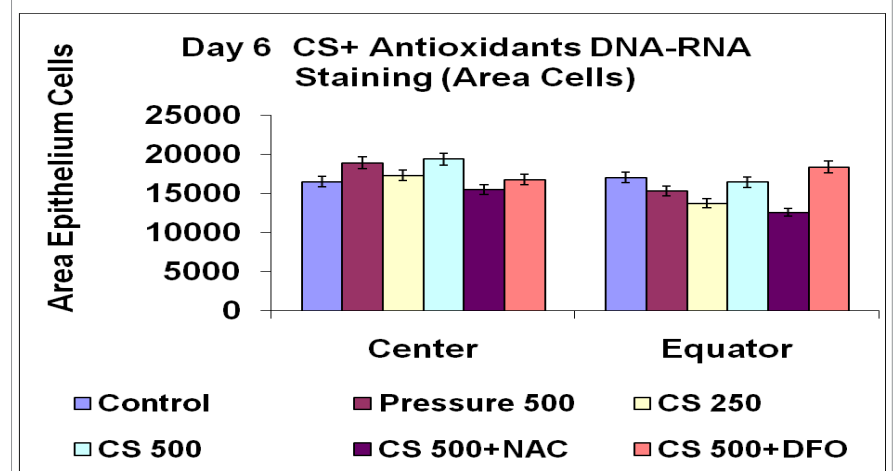

Figure 13: Area epithelium cells. DNA/RNA staining

\section{Detection of Oxidative Stress}

The antioxidants protective effect of each of the two agents were compared using the 5,6-chloromethyl-2',7'-dichloro- dihydrofluorescein diacetate (DCF) assay. Formation of ROS in the epithelium was monitored and detected, by fluorescence, in intact bovine epithelial cells layers, from the different treatment groups. Figures 14a and 14b shows a molecule of non-fluorescent (reduced and acetylated) DCF (Figure 14).

Note the low fluorescence of the epithelial cells (Figure 15).

Note the high fluoresce in the epithelial cells which indicate high oxidation (Figure 16).

NAC treated lens epithelium after 4 days in organ culture conditions. NAC prevents the oxidation in the cells and the cells looks like the controls. DFO reduced the oxidation levels in the cells. DFO slightly reduced the damage to the cells, while NAC totally prevents the damage. The epithelium from lenses treated with in the presence of NAC looks the same as the controls (Figure 17).

We have demonstrated a role for oxidative damage CS formation. This is in accord with previous proposals [18-20]. Thus, the possible use of antioxidant agents in cataract prevention and treatment is highly appealing. Future investigations should consider this possibility in human subjects. It is likely that NAC and DFO could provide a beneficial outcome. Sigarette smoke is a complex aerosol
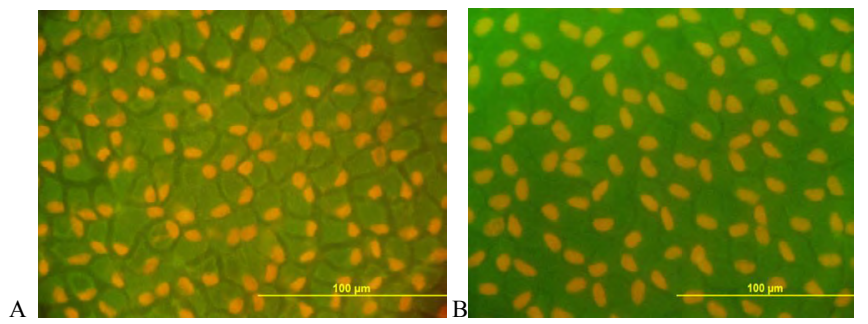

Figure 14: Control lens epithelium after 6 days in organ culture conditions $(A)$ and 4 from them with pressure medium $500 \mathrm{mmHg}(\mathrm{B})$.
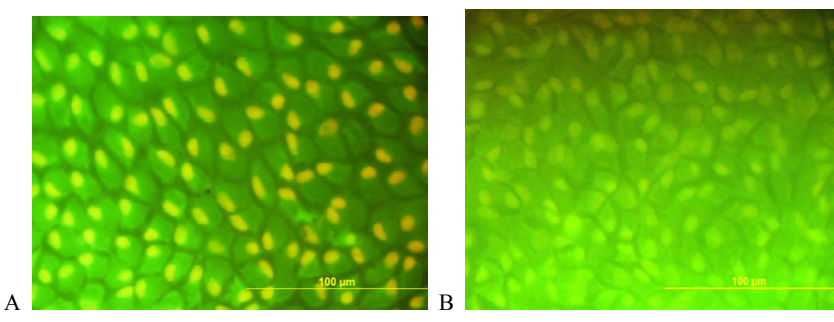

Figure 15: Epithelium lens after 6 days in organ culture and 4 from them with $\mathrm{CS}-250 \mathrm{mmHg}(\mathrm{A})$ and 4 from them with $\mathrm{CS}-500 \mathrm{mmHg}(\mathrm{B})$.
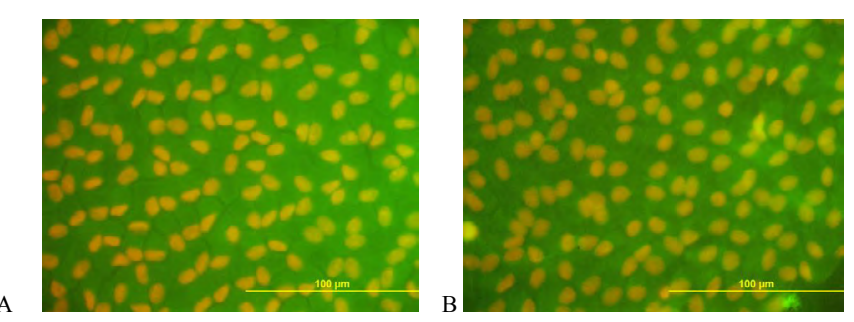

Figure 16: Epithelium lens after 6 days in organ culture and 4 from them with CS-500 mmHg+NAC(A). and 4 from them with CS-500 mmHg+DFO(B). 

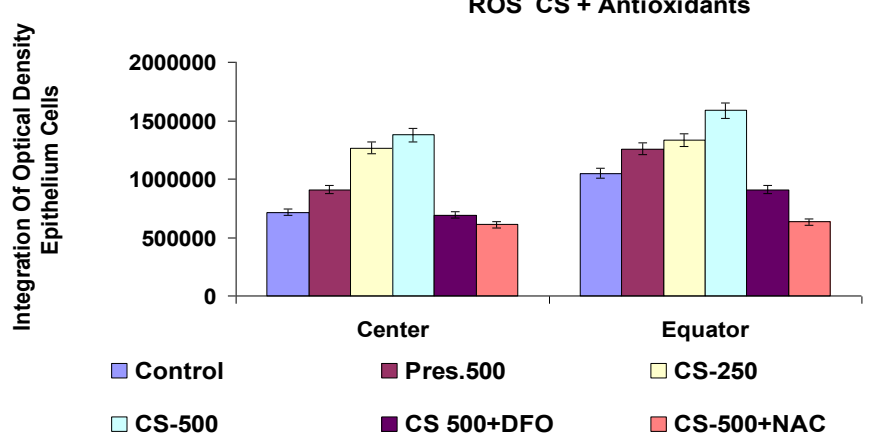

Figure 17: Integration of optical density epithelium lens. ROS

consisting of thousands of various constituents including reactive oxygen and nitrogen free radicals, toxic aldehydes and more. Previous epidemiological studies have identified tobacco smoking as a risk factor for sarcopenia. Clinical, in vivo and in vitro studies have revealed CSinduced skeletal muscle damage due to impaired muscle metabolism, increased inflammation and oxidative stress, over-expression of atrophy related genes and activation of various intracellular signaling pathways [21]. The a etiology of these conditions is thought to fit with the 'free radical theory' of ageing which postulates that ageing and agerelated diseases result from the accumulation of cellular damage from reactive oxygen species (ROS). Mitochondrial energy production is a major source of endogenous ROS. External sources of ROS include environmental sources especially solar radiation, biomass fuels and tobacco smoking. There is strong evidence from epidemiological studies that smoking is a risk factor for both cataract and AMD. There is moderate evidence for an association with sunlight and cataract but weak evidence for sunlight and AMD. The few studies that have investigated this suggest an adverse effect of biomass fuels on cataract risk. The antioxidant defence system of the lens and retina include antioxidants vitamins $\mathrm{C}$ and $\mathrm{E}$ and the carotenoids lutein and zinc, and there is mixed evidence on their associations with cataract and AMD from epidemiological studies. Add CART (cocaine and amphetamine regulated transcript) is a novel, brain-enriched mRNA which predicts a novel protein without homology to any known protein or peptide [22]. CART mRNA has been localized to ganglion cells of the retina, lamina $\mathrm{X}$ of the spinal cord, mitral and tufted cells of the olfactory bulb, barrel field neurons of the somatosensory cortex, the anterior pituitary, and the medulla of the adrenal cortex. The two alternatively spliced CART variants present in the rat brain were found to have identical and overlapping distributions in the rat forebrain. The retina contains ganglion cells (RGCs) that respond selectively to objects moving in particular directions. Individual members of a group of ON-OFF direction-selective RGCs(ooDSGCs) detect stimuli moving in one of four directions: ventral, dorsal, nasal, or temporal. Kay et al. [23] also identify a neuropeptide, CART (cocaine- and amphetamine-regulated transcript), that distinguishes all ooDSGCs from other RGCs. Together, this panel of endogenous and transgenic markers distinguishes the four ooDSGC subsets. Patterns of molecular diversification occur before eye opening and are therefore experience independent. . They demonstrate differences among subsets in their dendritic patterns within the retina and their axonal projections to the brain. Mao et al. [24] demonstrate that CART protects mitochondrial DNA (mtDNA), cellular proteins and lipids against the oxidative action of hydrogen peroxide, a widely used oxidant. Using cis-parinaric acid as a sensitive reporting probe for peroxidation in membranes, and a lipid-soluble azo initiator of peroxyl radicals, 2,2'-azobis(2,4-dimethylvaleronitrile) they found that CART is an antioxidant. Furthermore, they found that CART localized to mitochondria in cultured cells and mouse brain neuronal cells. Weiner et al. [25] study was to elucidate the effect of cigarette smoke (CS) on nitrotyrosine formation in saliva proteins. They exposed saliva to CS, in vitro, and used Western Blotting (WB) and monoclonal anti-nitrotyrosine antibody to assess the level of saliva protein nitration. As saliva contains extensive amounts of nitrites, it was no surprise that at basal levels, saliva proteins, albumin, and alphaamylase all were already nitrated. The WB also revealed that with continuous exposure to CS the tyrosine nitration of both albumin and $\alpha$-amylase is declining significantly after $3 \mathrm{~h}$. A quite similar effect was seen after exposure to aldehydes, but to a less extent as compared to CS. Exposure of nitrotyrosine-modified bovine serum albumin (BSA-N) to aldehydes, produced a similar effect, meaning a decrease in tyrosine nitration. Therefore, it is possible that the main player in the CS smoke denitration effect on salivary proteins is the aldehyde group and not the double bond of unsaturated aldehydes, masking the nitro group and thus not recognized by the antinierotyrosine antibodies. Terefore it is suggested that results from accumulation of changes caused by highly reactive oxygen-derived species (ROS), including free radicals, known to induce cell damage [26]. Continuous exposure of the lens to oxidative stress has been shown to lead to reduced lens clarity and cataract formation [27]. Since the epithelial layer contains the bulk of the metabolic enzymes, and since labile iron has been incriminated as a necessary factor for injurious oxidative stress, the damage to the epithelial layer can precede and account for the development of lens opacity [28]. Indeed, the levels of redoxactive iron were found elevated in the advanced forms of cataract [29]. The work done by Khanna et al. [30] have demonstrated that an $\mathrm{N}$-acetyl cysteine (NAC) treatment significantly reduced tobacco-exposed induced infarct size and percent fractional shortening. A significantly increased LV end-systolic diameter was observed in tobacco-exposed sham compared to tobacconaïve sham (4.92 \pm 0.41 vs $3.45 \pm 0.33 ; \mathrm{P}<0.05)$, and tobacco-exposed MI compared to tobacco-naïve MI $(8.24 \pm 0.3$ vs $6.1 \pm 0.49 ; \mathrm{P}<0.01)$ rats. Decreased intracardiac mRNA expression of the markers of inflammation, tissue repair and oxidative stress and circulating levels of pro-inflammatory cytokines accompanied these positive effects of NAC. The treatment of tobacco-exposed MI rats with NAC resulted in significantly increased levels of intracardiac mRNA expression of antioxidants, including superoxide dismutase, thioredoxin and nuclear factor-E2-related factor 2, as well as circulating levels of glutathione $(7 \pm 0.12$ vs $10 \pm 0.18$; $\mathrm{P}$-naïve sham rats. These findings identify a novel post-infarction therapy for amelioration of the adverse effects of tobacco exposure on the infracted myocardium and advocate the use of dietary supplement antioxidants for habitual smokers to prevent and reverse cardiovascular adverse effects. Avunduk et al. [31] made the conclusion that cataractogenesis after tissue influence of a smoke in rats was connected with iron accumulation, and concurrent deferoxamine(DFO) therapy prevented such cataract formation. Cigarette smoking is an important risk factor for cardiovascular, respiratory, and malignant diseases. There is also a strong association between smoking and a number of common eye diseases, which include Graves' ophthalmopathy, age-related macular degeneration, glaucoma and cataract [32]. Despite the multifactorial aetiology of these ocular syndromes, smoking is an independent risk factor that has dose-response effects. It causes morphological and functional changes to the lens.

\section{Conclusions}

We have shown that increasing the amount of smoke, for relatively short time, causes a sharp increase in the damage to the lens. Lenses 
Citation: Bormusov E, Reznick AZ, Dovrat A (2013) Potential Protection by Antioxidants of the Action of Tobacco Smoke on the Metabolism of Cultured Bovine Lenses. Metabolomics 3: 124. doi:10.4172/2153-0769.1000124

measured in focal length showed damage starting from about 48 hours after initial exposure to high concentrations CS. Lens intensity shows higher transparency as a result of the antioxidants treatment. Lens epithelium stained for nucleic acids show that $\mathrm{N}$-acetyl-L-cysteine reduced the damage to lens epithelial cells caused by high CS exposure. The results will help in understanding the mechanisms of damage to the eye lens by CS and whether oxidation is involved in the damage. Smoking is an independent risk factor that has dose-response effects. It causes morphological and functional changes to the lens. Based on the independent effects of NAC and DFO, we propose to use these agents as means of prevention and/or treatment of cataract associated with cigarette smoking.

\section{Acknowledgments}

The authors thank the Guzik Research Fund for supporting this study. The help and support of Prof. Lena Lavie is greatly appreciated.

\section{References}

1. Ambrose JA, Barua RS (2004) The pathophysiology of cigarette smoking and cardiovascular disease: an update. J Am Coll Cardiol 43: 1731-1737.

2. Lee J, Taneja V, Vassallo R (2012) Cigarette smoking and inflammation: cellular and molecular mechanisms. J Dent Res 91: 142-149.

3. Avunduk AM, Yardimci S, Avunduk MC, Kurnaz L, Koçkar MC (1995) Determinations of some trace and heavy metals in rat lenses after tobacco smoke exposure and their relationships to lens injury. Exp Eye Res 65: 417423

4. Fletcher AE (2010) Free radicals, antioxidants and eye diseases: evidence from epidemiological studies on cataract and age-related macular degeneration. Ophthalmic Res 3: 191-198.

5. Nagler R, Lischinsky S, Diamond E, Drigues N, Klein I, et al. (2000) Effect of cigarette smoke on salivary proteins and enzyme activities. Arch Biochem Biophys 379: 229-236.

6. Khanna AK, Xu J, Mehra MR (2011)Antioxidant N-acetyl cysteine reverses cigarette smoke-induced myocardial infarction by inhibiting inflammation and oxidative stress in a rat model. Lab Invest 92: 224-235

7. Avunduk AM, Yardimci S, Avunduk MC, Kurnaz L (1999) Cataractous changes in rat lens following cigarette smoke exposure is prevented by parenteral deferoxamine therapy. Arch Ophthalmol 117: 1368-72.

8. Reddan J, Sevilla M, Giblin F, Padgaonkar V, Dziedzic D, Leverenz V (1992) Tempol and deferoxamine protect cultured rabbit lens epithelial cells from $\mathrm{H} 2 \mathrm{O} 2$ insult: insight into the mechanism of $\mathrm{H} 2 \mathrm{O} 2$ - induced injury. Lens Eye Toxic Res 9: 385-393.

9. Atkuri KR, Mantovani JJ, Herzenberg LA, Herzenberg LA (2007) N-Acetylcysteine--a safe antidote for cysteine/glutathione deficiency. Curr Opin Pharmacol 7: 355-359

10. Dovrat A, Weinreb O (1995) Recovery of lens optics and epithelial enzymes after ultraviolet A radiation. Invest Ophthalmol Vis Sci. 36: 2417-2424.

11. Dovrat A, Weinreb O (1999) Effects of UV-A radiation on lens epithelial NaKATPase in organ culture. Invest Ophthalmol Vis Sci 40: 1616-1620.

12. Rom O, Kaisari S, Aizenbud D, Reznick AZ (2013) Cigarette smoke and muscle catabolism in C2 myotubes. Mech Ageing Dev 134: 24-34.

13. Dovrat A, Sivak JG (2005) Long-term lens organ culture system with a method for monitoring lens optical quality. Photochem Photobiol 81: 502-505.

14. Sivak JG, Yoshimura M, Weerheim J, Dovrat A (1990) Effect of hydrogen peroxide, DL-propranolol, and prednisone on bovine lens optical function in culture. Invest Ophthalmol Vis Sci 31: 954-963.

15. Faddeev AP (1962) Effect of X-ray radiation on an epithelium of lens animals Abstract. Odessa: 8-9.
16. Bormusova EA (1979) Histochemical characteristics of the lens epithelium of the rabbit eye in ontogenesis (oxyreductase, hidrolase): 494-497.

17. Pearse AG (1972) Histochemistry, Theoretical and Applied. (3 Edn), London: 753-1518.

18. Lou MF (2003) Redox regulation in the lens. Prog Retin Eye Res 22: 657-682.

19. Shin DM, Jeon JH, Kim CW, Cho SY, Kwon JC, et al. (2004) Cell type-specific activation of intracellular transglutaminase 2 by oxidative stress or ultraviolet irradiation: implications of transglutaminase 2 in age-related cataractogenesis. J Biol Chem, 279: 15032-15039.

20. Tarwadi K, Agte V (2004) Linkages of antioxidant, micronutrient, and socioeconomic status with the degree of oxidative stress and lens opacity in indian cataract patients. Nutrition 20: 261-267.

21. Rom O, Kaisari S, Aizenbud D, Reznick AZ (2012) Identification of possible cigarette smoke constituents responsible for muscle catabolism. J Muscle Res Cell Motil 33: 199-203.

22. Couceyro PR, Koylu EO, Kuhar MJ (1997) Further studies on the anatomica distribution of CART by in situ hybridization. J Chem Neuroanat 12: 229-241.

23. Kay JN, De la Huerta I, Kim IJ, Zhang Y, Yamagata M (2011) Retinal ganglion cells with distinct directional preferences differ in molecular identity, structure and central projections. J Neurosci 31: 7753-7762.

24. Mao $P$, Meshul CK, Thuillier $P$, Goldberg NR, Reddy PH (2012) CART peptide is a potential endogenous antioxidant and preferentially localized in mitochondria. PLoS One 7

25. Weiner D, Khankin EV, Levy Y, Aizenbud D, Reznick AZ (2010) Effects of cigarette smoke on salivary protein tyrosine nitration. Eur J Med Res 15: 211 216.

26. Rosini M, Andrisano V, Bartolini M, Bolognesi ML, Hrelia P, et al. (2005) Rational approach to discover multipotent anti-Alzheimer drugs. J Med Chem 48: $360-363$

27. Marsili S, Salganik RI, Albright CD, Freel CD, Johnsen S, et al. (2004) Cataract formation in a strain of rats selected for high oxidative stress, Exp Eye Res 79 : 595-612.

28. Giblin FJ, McCready JP (1983) The effect of inhibition of glutathione reductase on the detoxification of $\mathrm{H} 2 \mathrm{O} 2$ by rabbit lens. Invest Ophthalmol Vis Sci 24 113-118.

29. Garner B, Davies MJ, Truscott RJ (2000) Formation of hydroxyl radicals in the human lens is related to the severity of nuclear cataract. Exp Eye Res 70 : 81-88.

30. Khanna AK, Xu J, Mehra MR (2011) Antioxidant N-acetyl cysteine reverses cigarette smoke-induced myocardial infarction by inhibiting inflammation and oxidative stress in a rat model. Lab Invest 3: 146.

31. Avunduk AM, Yardimci S, Avunduk MC, Kurnaz L (1999) Cataractous changes in rat lens following cigarette smoke exposure is prevented by parenteral deferoxamine therapy. Arch Ophthalmol 117: 1368-1372.

32. Cheng AC, Pang CP, Leung AT, Chua JK, Fan DS, et al. (2000) The association between cigarette smoking and ocular diseases. Hong Kong Med J. 6: 195-202. 